\title{
Recovery and resilience: how can innovation policy support the response
}

\author{
Jonathan Cook and Tim Vorley
}

\section{Introduction}

Innovation is widely regarded as key to driving economic growth and raising productivity. For many firms, large and small, looking to survive the economic downturn that has accompanied the COVID-19 crisis, their immediate priority has been cost saving. The level of private investment in innovation will likely fall in the wake of the pandemic as firms struggle to ensure the viability and continuity of their operations. However, innovation and innovative firms will be critical to the recovery of the economy in the UK and elsewhere. This presents a paradox in one of the latest twists in the productivity puzzle: a need for more private investment in innovation at a time when it is at risk of declining, Moreover, the uneven geography of innovation in the UK would appear to suggest that the North of England, Wales and Northern Ireland will be hardest hit. Yet it is these regions which are amongst the least innovative in the UK.

Since the Budget in March 2020 and during the subsequent lockdown, the Government has announced and implemented measures to try and mitigate economic impacts of the pandemic. Alongside the various general mechanisms to support businesses, there have been and continue to be specific measures to support and sustain innovation. The effectiveness of such interventions cannot be gauged on their uptake in the short term, but rather innovation outcomes associated with the UK's post-COVID recovery in the medium term and beyond. As such the role of innovation policy at this point is critical to sustaining innovative activity, ensuring the survival of innovative firms and supporting their future success. Under any circumstance this is a big ask, let alone in the wake of a pandemic when uncertainty is rife.

In 2017, some 10 years after the Great Financial Crisis, the Government's response to the UK's flat-lining productivity was a white paper setting out its flagship Industrial Strategy (HM Government, 2017). A central tenet of the Industrial Strategy was to increase innovation in order to help drive productivity across the UK. The Industrial Strategy Challenge Fund saw an initial investment of $£ 725 \mathrm{~m}$ allocated for programmes to capture the value of innovation. These programmes sought to encourage collaboration amongst businesses and between industry and the research base. Increased collaboration in research, science and innovation can bring about spillovers and positive externalities, and 
is seen as key to addressing societal challenges, including the grand challenges (AI and Data, Ageing Society, Clean Growth, and the Future of Mobility).

A core objective set by the Industrial Strategy was to raise spending on R\&D to reach the OECD average of $2.4 \%$ of GDP by 2027. This will require increased public investment, and as well as commitments in the Industrial Strategy there have been more recent announcements, including the intention to establish an Advanced Research Projects Agency (modelled on the defence version in the USA). It will also require leveraging more private investment, including into earlier stage R\&D that includes technologies that may be slower to market. As Bailey and Tomlinson (2017) suggest, the Industrial Strategy is about addressing market failures and enabling 'winners' to emerge rather than picking them. The crux of the Industrial Strategy is as much innovation policy as it is industrial policy, with the emphasis on the advent of new technologies, and with it enhancing and transforming innovation trajectories (Bailey et al., 2019). The recent R\&D Roadmap sets a vision for the UK in terms of science and R\&D and highlights the need to identify and address strengths and challenges in R\&D so that innovation can play a critical role in the economic and social recovery from COVID-19 (HM Government, 2020).

An inherent challenge of innovation policy identified by Kitson (2019) is the tendency to emphasise the generation of innovations as opposed to the diffusion and adoption of innovations. It is noteworthy that the Industrial Strategy recognises the need to improve the diffusion and scaling of innovation (HM Government, 2017), as well as the pursuit and development of new technologies. While innovation can be considered a key driver of productivity, the importance of diffusion and scaling the use of innovations needs to be recognised as critical to future productivity growth. This is particularly the case among small and medium-sized enterprises (SMEs) so that they can access and use tools that can help them become more productive. Some actions are in place to support diffusion. The Government's 'Business Basics' fund is trialling and evidencing new approaches to sharing good practice, while local Growth Hubs bring public and private partners together to ensure local businesses have access to support and serve to promote technological adoption.

This chapter considers how COVID-19 may affect key drivers of innovation, and reflects on how the UK's policy frameworks could be adapted to aid the response to the crisis. There is of course uncertainty concerning how economic, societal, political and technological will evolve, and the discussion is based on what we know and anticipate at this point. In discussing innovation and the pandemic the chapter is structured in three sections, namely: 1) the adoption and diffusion of new technologies and new practices; 2) R\&D investment and the role of collaboration; and 3) the mix and focus of policy.

\section{Adoption and diffusion of new technologies and practices}

In response to the pandemic, businesses have been substantially occupied with issues around cash and survival, furloughing staff, accessing relevant government support, and when and how they may be able to reopen. Another short-term response that may have 
longer-term implications for innovation is the adaptation of businesses. There are different aspects to this, and two that have been apparent in responses to the crisis are the related areas of: adopting new working practices, which have included the use of technology; and product, service and/or business model innovation, again in some cases facilitated by technology.

Driven by necessity, many firms have responded to the pandemic through changes in their working practices. For firms that have been able to do so, this has involved the use of remote working and teleconferencing. The British Chambers of Commerce Business Impact Tracker survey of 600 companies indicated that the majority of respondents had embraced the change in working practices, with 66 per cent of respondents using remote working and 50 per cent teleconferencing (Crapnell, 2020). FSB research showed that 30 per cent of small businesses had reported that they had altered their business practices to accommodate working from home (Rustamova, 2020).

A key enabler of this has been the use of digital technologies. Those already digitallyenabled were able to respond more quickly, whilst other firms have adopted new technologies. The FSB research indicated that 24 per cent of the small firms that they surveyed had increased or adopted new digital technologies such as online communications technologies to facilitate working from home (Rustamova, 2020). For the most part these are not 'advanced technologies' but are instead basic or general-purpose technologies that are used in more advanced ways to enable (remote) collaboration and connectivity. While enabling firms to maintain, if not improve their productivity, these technologies predominantly serve to perpetuate existing ways of working at a distance as opposed to invoking new ways of working per se. In addition, though much attention has been placed on these kinds of general purpose technologies, there has also been adoption of technology to change ways of working. For example, technology has altered working practices in some settings, such as the use of robots to carry out tasks to protect humans and enable social distancing, e.g. cleaning in common areas of buildings.

Increased confidence in using technology, driven initially by necessity, together with concerns over ensuring resilience of businesses in relation to finance, people, markets and operations, may contribute to longer-term changes in attitudes towards innovative practice and technology. This may in part be driven by employee expectations, for instance as more people expect to be able to work more flexibly in the future. Employers themselves may also see the business benefits, for instance in terms of recruitment, retention and staff well-being, and reduced needs for office space. These benefits may lead to productivity enhancements, which may also arise from the use of other technologies such as robots. A number of employers have already signalled longer-term or even permanent shifts towards more home-working, e.g. Mastercard, Google, Facebook and Twitter.

The crisis has also led to pivoting and business model changes as businesses have sought to respond to a need to serve their customers in different ways and through the development of new or adapted products/services. Again, technology has facilitated this, with firms as well as other organisations such as those in healthcare (for video 
appointments) and education (through online provision of learning) all using online channels. The digital capability of businesses has come to represent a key component of their resilience, both in terms of their own operations as well as the ability of firms to offer their products and services through alternative channels.

New drivers of markets create opportunities for innovators and entrepreneurs to develop new products and services, or new ways of delivering products and services. Isenberg and Schultz (2020) have identified a series of enduring drivers that could create new opportunities in the "coronomy", including in the medium-term. These drivers include remote interaction, the centrality of the home, and how goods are distributed; and this has created new or greater opportunities associated with collaboration platforms, telemedicine, remote learning, and the use of robotics and drones. There are some important overlaps in these enduring drivers and the opportunities that are associated with them, including the role of digital, assistive and other technologies and the potential to contribute to societal objectives through supporting resilience for businesses and people. These are discussed later in this chapter in relation to policy priorities and objectives.

The short-term responses described above are positive. Indeed, taking advantage of changes in attitudes towards technology use and working practices, and the long-term trends that could be stimulated, could support improvements in levels of performance on productivity. However, there are some significant challenges that need to be overcome. Previous evidence on the take up of technologies, including digital technologies and robotics, and on investment in capital has shown that the UK has performed relatively poorly compared to international comparators.

The Made Smarter Review highlighted the UK's relative weaknesses in the use of robots, with the UK having only 33 robots per 10,000 employed people compared to 93 for the US and 170 for Germany with the gap widening (Maier, 2017). The Review also noted the higher levels of investment in automation in countries such as Germany, and that Germany and Japan have higher numbers of robots per millions of hours worked by a factor of more than 10 than the UK (Maier, 2017). A report by McKinsey Global Institute found that the UK performs well on some of the more basic digital adoption issues such as internet access, basic digital skills and cloud computing. However, it performs poorly on more sophisticated aspects such as the integration of information systems across the value chain, business process transformations, enterprise digitisation and robotics (McKinsey Global Institute, 2018).

The barriers that are preventing higher levels of take-up of technologies amongst UK companies need to be addressed. Key barriers to adoption of digital technologies and robotics include risk aversion, skills shortages, finance, and the requirements for managerial and business model change (McKinsey Global Institute, 2018; Maier, 2017). Changing working practices will also require new skills and effective management and leadership if they are to be implemented effectively. 
These barriers and new requirements are potentially quite significant challenges. Whilst the appetite and interest in technology adoption, business model innovation and changes to working practices may have certainly increased as a result of the pandemic, skills, finance and managerial capacity will remain key barriers unless addressed. Skills, including leadership and management skills, were a key area recently flagged as being a risk to the UK economy over the next decade (Industrial Strategy Council, 2019).

In order for the UK to capitalise on this recent interest and the ongoing opportunities in relation to technology use, adoption of new working practices and business model innovation, there needs to be a change in the balance of policy intervention. Innovation policy in the UK has, in the last decade, been centralised (Cook et al., 2020) and focussed on frontier technology development. Whilst frontier technology development is needed, a key implication is that in supporting and accelerating the adoption of new technologies and practices, there needs to be more emphasis in innovation policy on diffusion.

\section{$R \& D$ investment, the $2.4 \%$ target and the role of collaboration}

Overall levels of R\&D and innovation activity were adversely affected by the 2008 financial crisis (Roper, 2020). Within this, there was significant variation with the speed of recovery in R\&D after the financial crisis varying geographically and sectorally. Following the Great Financial Crisis public sector R\&D spending was more resilient than overall economic activity, although business investment was more adversely affected. Levels of business investment in R\&D were uncertain, and the challenge facing smaller firms to sustain R\&D spending was particularly acute. Lee et al. (2015) also highlighted the structural problem which restricts access to finance for innovative firms. There is a range of evidence indicating that $R \& D$ and innovation activity is procyclical, and that government-financed R\&D has a modest countercyclical effect. This is not entirely surprising given the role of public funding to stimulate demand, and specifically around 'mission-oriented' innovation as is required to realise the objectives associated with the Industrial Strategy's Grand Challenges.

Prior to the pandemic, analysis found higher levels of R\&D expenditure in the UK to boost GDP, employment and productivity outcomes albeit with a time lag (Pollitt et al., 2020). In the March 2020 budget, at the advent of the crisis in the UK, there were a number of measures to mitigate against the economic and social impacts of COVID-19. Subsequently the Government has further demonstrated its commitment to the target to invest $2.4 \%$ of GDP in R\&D by 2027. Despite this reaffirmed commitment, the pandemic has disrupted and distracted from research and development in business as well as across universities and research institutes. There is a real concern that this disruption could lead to an innovation gap where innovative companies are unable to access funding and survive given the known challenges around cashflow. To help address the prospect of short-term failures, the UK government's Future Fund was set up to support innovative firms if government funding was matched by private investors. 
It is unsurprising that private investment through equity into companies is beginning to be affected by COVID-19. Investors are becoming more cautious, and we have seen slowdown in deal flow and a reorientation of investment priorities and portfolios as firms adapt to a new economic reality. Clearly the volatility of external markets has impacted the willingness and ability of some investors to raise investment, and it is likely to be earlier stage companies and deep technology companies with longer and more uncertain routes to markets to be most affected. It is, however, the same so-called deep or frontier tech firms that could represent a key component of the $2.4 \%$ target (e.g. see SQW, 2020) and are most likely to drive the longer-term economic recovery. The challenge for innovation policy is ensuring the uncertainty caused by the COVID-19 pandemic does not see these future prospects go without the necessary investment and so undermine future growth potential.

Further to the importance of deep tech, which are highly concentrated in key centres (i.e. London and the Golden Triangle), there is a need to take a UK wide approach towards realising the $2.4 \%$ target. The 'Excellence Principle' both reinforces and is reinforced by the regional concentration of R\&D investment, and is testament to how the advantages of agglomeration and specialisation are manifest in place (McCann and Ortega-Argiles, 2015). Such concentrations of economic activity and resources can see clusters of R\&D activity become self-sustaining. However, there are few if any places outside of the Southeast in the UK that have been able to realise the benefits of agglomeration despite the growing focus on innovation districts and their equivalents.

Maximising the return on R\&D spending is an important factor, but this does not need to be mutually exclusive with more equitable regional distribution of R\&D spending. The CaSE (2020) report on the 'Power of Place' emphasises the need for investment to focus on existing centres of R\&D excellence; although to maximise the return on investment the focus needs to be on the ' $D$ ' not just the ' $R$ ' that already exists. This demands a greater emphasis on translational research and commercialisation, as well as strengthening industrial R\&D. This will not only contribute towards realising the $2.4 \%$ target, but see greater returns realised from R\&D investments.

In the pursuit of innovation there are well recognised benefits of R\&D collaborations (Belderbos et al., 2015), including increasing the value and productivity of R\&D activities. In addition, collaborative $R \& D$ can serve to reduce the financial risk to partners, which is important given that innovation projects facing financial barriers are more likely to fail or be abandoned (Mohnen et al., 2008). These benefits were demonstrated by the collaborative response of many organisations in the midst of the pandemic to the Ventilator Challenge, which saw manufacturers and medical device companies design new ventilators from scratch.

The challenge for innovation policy is to see the possibilities demonstrated in response to the crisis incorporated more into business as usual. It acknowledged that granting higher subsidies to collaborating firms has previously served to stimulate further R\&D spending (Bianchi et al, 2019). While there is likely to be increased demands for public sector funding for innovation, it is imperative that this is not for sustaining existing 
(collaborative) innovation projects, but to support new opportunities for recovery and growth. Such funding may well reflect different objectives and priorities compared to preCOVID funding and present an opportunity to recalibrate. Policy should also maintain a focus on non-financial support, which is critical to help innovative businesses access the advice required to enable them to reposition, grow and scale.

\section{Policy mix and priorities}

In itself the pandemic does not necessitate a major shift in innovation policy. That said, COVID-19 represents a juncture at which to review the objectives of innovation policy and to reflect as to whether the focus and balance needs to change. The pandemic has brought the economic and societal objectives of innovation policy into focus. As a health crisis, the pandemic has highlighted the varying degrees of vulnerability of different groups in society. It has reminded us that health is a public good, and that the lack of adequate infrastructure for healthcare can bring about huge economic and social costs (Lucchese and Pianta, 2020).

A further dimension brought into focus is the environment. The environmental benefits of the pandemic through the lockdown imposed on much of the world's population have been visible. Environmental pollution has reduced by up to 30\% (Muhammad et al., 2020), with potential knock-on effects on air quality, health and quality of life. This has given an insight into what a possible future might look like where climate change is more effectively addressed.

COVID-19 has also shown how interdependent global supply chains are, and the risks associated with this. Going forward, companies are likely to want to operate supply chains with more geographic sources and options to enhance their resilience. This will be a tradeoff for companies between short term profit maximisation and longer-term risk reduction (Wilson, 2020). There are also potential societal benefits if there is greater security of supply of key goods such as food and medicines.

Therefore, the pandemic provides an opportunity for society to rethink the balance of its objectives between, for instance, growth and prosperity, health and well-being, societal resilience, and the environment. The role of government will need to be substantive in financing, through taxation and deficit spending, a range of activities including health, education, research, social assistance, the environment and economic development. Innovation policy is key to this, and R\&D budgets are required to target issues such as heath systems, vaccine development and global challenges like climate change (te Velde, 2020).

The role of innovation in delivering objectives beyond growth and prosperity is not new. Whilst economic growth and productivity are often seen as the core focus, with three of Innovate UK's five objectives focused on business, industry growth and commercial impacts (Innovate UK, 2019), there has been an increasing focus on wider economic and societal objectives. One of Innovate UK's objectives relates to delivering economic and societal impact, aligning with UK Research and Innovation's three pillars of economy, 
society and knowledge. The Industrial Strategy Challenge Programme is fundamentally organised under economic and societal challenges, including healthy ageing and green growth. Further afield, other nations have similar multi-purpose objectives in relation to innovation and research, which is also exemplified through the Horizon Europe programme (European Commission, 2018).

In order for innovation policy to be effective in contributing to achieving economic and societal objectives, it is important to draw on innovation systems thinking. This requires consideration of a range of issues, including R\&D, networks and interactive learning of those involved in the innovation process, competence building, demand-side requirements from the perspective of product/service use, and finance for both commercialisation and adoption (Edquist, 2006). Now, more than ever, the wider merits and benefits of pro-active innovation policies are both apparent and necessary.

Figure 1 identifies four objectives of innovation policy, including the more conventional focus on 'Growth and Prosperity', as well as more societally-oriented objectives linked to the environment, health and well-being, and security and resilience. Seven enablers are set out in Figure 1: three are specifically within the remit of innovation policy and reflect on points identified in this chapter; and four are wider enablers. The issues associated with the three innovation policy enablers are summarised as follows:

- Investment: The balance of focus on economic and societal outcomes have implications for how and where investments are directed, in particular grant schemes. For instance, the four objectives set out in Figure 1 would result in a focus on key sectoral and technological priorities, such as medical, assistive, energy, transport, food and digital. Overlaying this, R\&D and other support for investment would need to reflect areas of acute financial shortages, e.g. stage, sector/technology and place. Such a prioritisation could represent an opportunity to recalibrate funding towards sectors and objectives where market failures are greatest.

- Diffusion and adoption: In order to achieve economic and societal objectives, there needs to be adoption of innovation. This needs to be society-wide: by households to ensure that they can benefit from aspects such as assisted living, preventative healthcare or digital services; by businesses to ensure that they are competitive, productive and resilient; and by other organisations to ensure that innovative services can be delivered, and in innovative ways. As highlighted earlier in the chapter, this area has received much less focus in innovation policy in the UK. This also links to other wider enablers set out in Figure 1, notably skills and capabilities in order to implement and use innovations (as well as develop them) and citizen engagement.

- Collaboration and networks: The inter-linkages between industry and the knowledge base to develop new ideas are critical, and this will require inter-disciplinary collaboration. This goes with the grain of UKRI policy and programmes. If innovation is to reflect more focus on societal objectives and be more attuned to encouraging adoption and behaviour change, there is a need for greater co-development of 
innovative products and services with users and communities. This could align with policies aimed at economic recovery, because system-wide policy design could link together innovation investment, co-development, adoption and demand-side incentives (such as incentives for green products and services). Looking more broadly, this will also need to link into retraining and skills (e.g. for new/more 'green' jobs) and infrastructure (e.g. digital, vehicle charging and energy infrastructure).

\section{Figure 1: Linking innovation policy to economic and societal objectives}

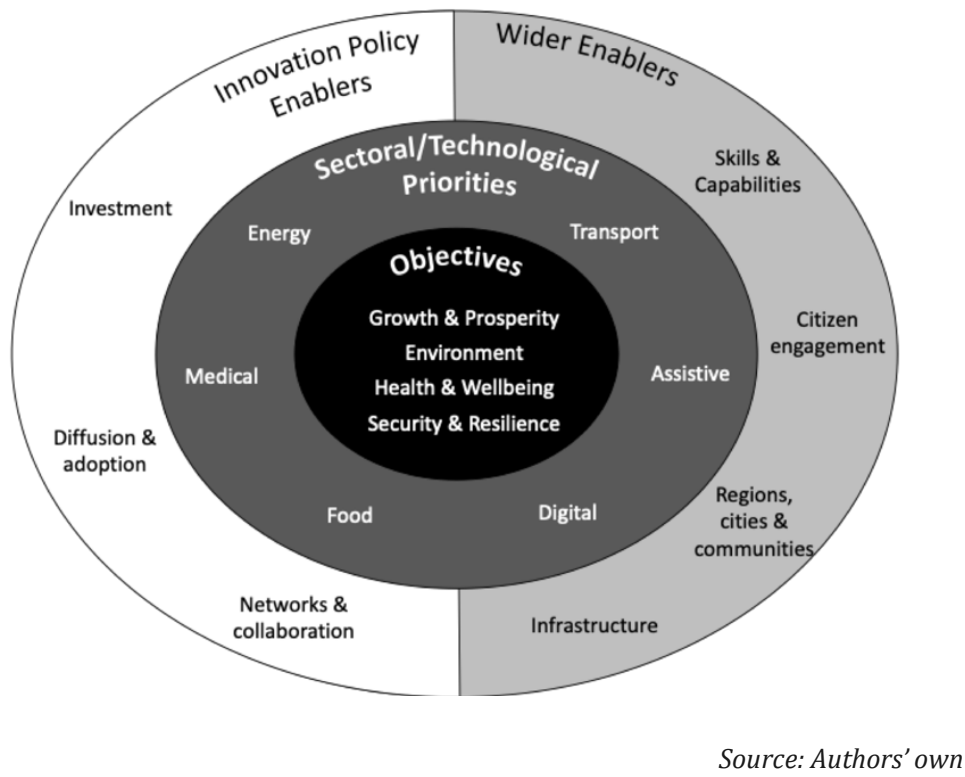

The framework for thinking about innovation policy outlined above has important implications for the nature of policy outcomes, how they would be measured and how the effectiveness of policy should be evaluated. In recent years, whilst there has been greater emphasis on mixed methods and consideration of assessing behavioural effects at the level of innovation systems (Weresa et al., 2018), the focus in the UK has been on economic growth (and associated indicators such as commercial impacts), particularly when assessing value for money. Economic growth and productivity effects may be an intended effect for certain interventions; for others, there may be greater emphasis on other outcomes such as behavioural change, adoption of new approaches or diffusion of practice to previously unreached groups, and ultimately outcomes such as health and the environment. This poses challenges to evaluation, measurement and demonstrating value for money, because system level effects are often harder to trace through and attribute to interventions and because these other outcomes cannot be monetised as readily. The pandemic has highlighted the opportunity, and need, for innovation policy to change. Therefore, it will be important for policy makers to reconsider the outcomes of innovation policy, and rethink the balance between economic and societal objectives. 


\section{References}

Bailey, D. and Tomlinson, P. R. (2017) Back to the future? UK industrial policy after the great financial crisis. In P. Arestis and M. Sawyer (eds) Economic Policies since the Financial Crisis, Palgrave MacMillan, Basingstoke, pp. 221-264.

Bailey, D., Glasmeier, A., Tomlinson, P.R., and Tyler, P. (2019) Industrial policy: new technologies and transformative innovation policies?, Cambridge Journal of Regions, Economy and Society, 12 (2) Pages 169-177, https://doi.org/10.1093/cjres/rsz006

Bianchi, M., Murtinu, S., \& Scalera, V. G. (2019). R\&D subsidies as dual signals in technological collaborations. Research Policy, 48(9), 103821.

CaSE (2020) The Power of Place: Maximising local economic impacts of R\&D investment in the UK, Campaign for Science and Engineering, London.

Belderbos, R., Carree, M., Lokshin, B., \& Sastre, J. F. (2015). Inter-temporal patterns of R\&D collaboration and innovative performance. The Journal of Technology Transfer, 40(1), 123137.

Clark, D. (2020) "Number of jobs furloughed under the job retention scheme in the UK 2020", www.statista.com. Available at https://www.statista.com/statistics/1116638/uknumber-of-people-on-furlough/ [Accessed $4^{\text {th }}$ August 2020]

Cook, J., Hardy, D. and Sprackling, I. (2020). Productivity Policy Review in McCann, P. and Vorley, T. (2020) Productivity Perspectives, Edward Elgar.

Crapnell, R. (2020), "BCC Coronavirus Business Impact Tracker: First results show heavy toll on UK business communities as majority of firms face cash flow crisis", www.britishchambers.org.uk. Available at https://www.britishchambers.org.uk/news/2020/04/bcc-coronavirus-business-impacttracker [Accessed 9th June 2020]

Edquist, C. (2006). "Systems of Innovation: Perspectives and Challenges" in Fagerberg, J. and Mowery, D.C. (2006) The Oxford Handbook of Innovation, Oxford University Press.

European Commission (2018). Proposal for a decision of the European Parliament and of the Council on establishing the specific programme implementing Horizon Europe - the Framework Programme for Research and Innovation. Brussels

HM Government (2017). Industrial Strategy White Paper: building a Britain fit for the future.

HM Government (2020). UK Research and Development Roadmap.

Industrial Strategy Council (2019). UK Skills Mismatch in 2030. Research Paper. 
Innovate UK (2019). Delivery Plan 2019. UK Research and Innovation.

Isenberg, D. and Schultz, E.B. (2020), “Opportunities for Entrepreneurs in the Pandemic and Beyond", www.medium.com. Available at https://medium.com/@disen2/opportunitiesfor-entrepreneurs-in-the-pandemic-and-beyond-f92f5fa1997b [Accessed 9th June 2020]

Kitson (2019). Innovation policy and place: a critical assessment. Cambridge Journal of Regions, Economy and Society, Volume 12, Issue 2, July 2019, Pages 293-315

Lee, N., Sameen, H., \& Cowling, M. (2015). Access to finance for innovative SMEs since the financial crisis. Research Policy, 44(2), 370-380.

Lucchese, M. and Pianta, M. (2020) "Coronavirus crisis: major economic and financial consequences", $\quad$ www.opendemocracy.net. Available at https://www.opendemocracy.net/en/can-europe-make-it/economic-consequencescoronavirus-major-economic-and-financial-crisis/ [Accessed 12 ${ }^{\text {th }}$ June 2020]

Maier, J. (2017). Made Smarter Review.

McCann, P. and Ortega-Argilés, R. (2015) Smart specialisation, regional growth and applications to European Union cohesion policy, Regional Studies, 49: 1291-1302.

McKinsey Global Institute (2018). Solving the United Kingdom's Productivity Puzzle in a Digital Age. Discussion Paper.

Mohnen, P., Palm, F., van der Loeff, S. and Tiwari, A. (2008): "Financial Constraints and Other Obstacles: are they a Threat to Innovation Activity?", De Economist, 156: 201-214.

Muhammad, S., Long, X., and Salman, M. (2020) “COVID-19 pandemic and environmental pollution: A blessing in disguise?" in Science of the total environment, Vol 728.

Pollitt, H., Chewpreecha U. and Berg von Linde, M. (2020) Macroeconomic modelling of the 2.4\% R\&D target, BEIS Research Paper Number 2020/009

Roper, S. (2020), R\&D and innovation after Covid-19: What can we expect? A review of trends after the financial crisis. ERC Insight Paper

Rustamova, C. (2020). New Horizons: How Small Firms are Navigating the COVID-19 Crisis. Federation of Small Businesses.

SQW (2020), Assessment of the economic and wider benefits of the UK Innovation and Science Seed Fund. Final report to Midven Ltd on behalf of the UKI2S Partners.

Te Velde, D. W. (2020), "The economic impact of coronavirus: five lessons and challenges", www.odi.org. Available at https://www.odi.org/blogs/16764-economic-impactcoronavirus-five-lessons-and-challenges [Accessed $12^{\text {th }}$ June 2020] 
Weresa, M., Poel, M., Cunningham, P. and den Hortog, P. (2018), Evaluation of Business R\&D Grant Schemes: behavioural change, mixed-method approaches and big data, Final report of the Mutual Learning Exercise under the Horizon 2020 Policy Support Facility (PSF), European Commission: Brussels

Wilson, J. (2020), "The economic impact of coronavirus: analysis from Imperial experts", www.imperial.ac.uk. Available at https://www.imperial.ac.uk/news/196514/theeconomic-impact-coronavirus-analysis-from/ [Accessed $12^{\text {th }}$ June 2020] 